\title{
CHARACTERIZING PROTON BEAM OF 6.7 MeV LEDA RFQ BY FITTING WIRE-SCANNER PROFILES TO 3-D NONLINEAR SIMULATIONS*
}

\author{
W.P. Lysenko ${ }^{\dagger}$, J.D. Gilpatrick, J. Qiang, L.J. Rybarcyk, R.D. Ryne ${ }^{\ddagger}$, J.D. Schneider, H.V. Smith, \\ L.M. Young, LANL, Los Alamos, NM 87545, USA \\ M.E. Schulze, General Atomics, Los Alamos, NM 87544, USA
}

Abstract

Quadrupole scans were used to characterize the transverse beam of the LEDA RFQ. The original scan data, obtained when the HEBT was installed immediately downstream of the RFQ, were reanalyzed by fitting to the 3-D nonlinear simulation code IMPACT. All the data in the measured profiles at the wire scanner were used, not just the rms widths. The measured and simulated profiles still have differences in their shapes that have not been explained. An improved model is required to get better fits and may result in more accurate emittance measurements.

\section{INTRODUCTION}

Analyses of quadrupole-scan data to determine transverse proton beam properties of the $6.7 \mathrm{MeV}$ LEDA RFQ have been presented[1,2]. In these experiments, there were four quadrupoles in the HEBT between the RFQ exit and the beam stop. The gradient in one of the two quadrupoles just downstream of the RFQ was varied while the beam was observed at the wire scanner near the end of the HEBT. Profiles were recorded for various values of the gradient. For an $x$ scan, Q2 was varied. For a $y$ scan, Q1 was varied. In both cases, Q3 and Q4 were off. As the gradient of the varied quad was increased, the beam size, which started with a large value, decreased to some minimum and then increased again. To determine the Courant-Snyder parameters of the beam at the RFQ exit, we fit the rms beam size as a function of the quadrupole gradient to TRACE 3-D and LINAC code simulations. The TRACE 3-D envelope (moment) code includes 3-D space-charge effects but not nonlinearities, while the particle code LINAC includes nonlinear space-charge effects but is $r-z$ (not 3-D).

In the present work, we fit the same experimental data to the IMPACT code, which is both nonlinear and 3-D. Another new feature of the present work is that the fits minimize the error in the detailed shapes of the wire-scanner profiles, not just their rms widths.

\footnotetext{
${ }^{*}$ Supported by US DOE, Office of Defense Programs and the Office of Nuclear Energy, Science and Technology.

†.wpl@lanl.gov

$\ddagger$ present address: LBNL, Berkeley, CA 94720, USA
}

\section{PREDICTED BEAM AND RMS FIT TO LINAC CODE}

All graphs in this paper compare the measured profiles at the wire scanner with IMPACT simulations that transport the beam from the RFQ exit to the wire-scanner location. The different cases correspond to different initial beams (beams at the RFQ exit). Figure 1 compares the measured profiles with IMPACT simulations using as input the RFQ output beam predicted by the PARMTEQM RFQ simulation code. The top row shows profiles for three values of Q2 for the $x$ scan and the bottom row shows profiles for three values of Q1 for the $y$ scan. In both cases, the graph in the middle shows the profile having a width near the minimum.

For gradients larger than a certain value (near beam size minimum), the measured profiles had a shape consisting of a triangular base (tail) and a narrow core. For these beams, there is a waist upstream of the wire scanner. The simulated profiles did not exhibit this change in shape for higher quad strengths.

Figure 2 compares the measured profiles with IMPACT simulations using as input the RFQ beam determined by fitting the rms widths to LINAC code simulations[2]. In the $x$ direction, the profiles for the rms LINAC fit are similar to the PARMTEQM predictions, although the fitted emittance is larger than the prediction, as shown in Table I. For the $y$ direction, the agreement for the fitted profiles is improved compared to the PARMTEQM prediction and the emittance is again larger than predicted.

\section{FITTING TO IMPACT CODE}

In fitting to the nonlinear 3-D IMPACT code, we used all the profile data, not just the rms widths. For the $x$ scans, for each of the 11 values of Q2 and for each of the $51 x$ positions of the wire, the difference between the measured intensity and the simulated intensity at the wire position was computed. It is the sum of the squares of these 561 differences that is minimized by varying the values of $\alpha_{x}$, $\beta_{x}$, and $\epsilon_{x}$ of the input beam (beam at RFQ exit).

Figure 3 compares the measured profiles with IMPACT simulations using as input the best fit to the IMPACT model. better than either the prediction (Fig. 1) or the rms LINAC fit (Fig. 2). For the larger gradient values, we still do not have the small core and large triangular tail seen in the measurements. As seen in Table I, the emittance values for this IMPACT fit (labeled IMPACT fit 1) are smaller than for the 

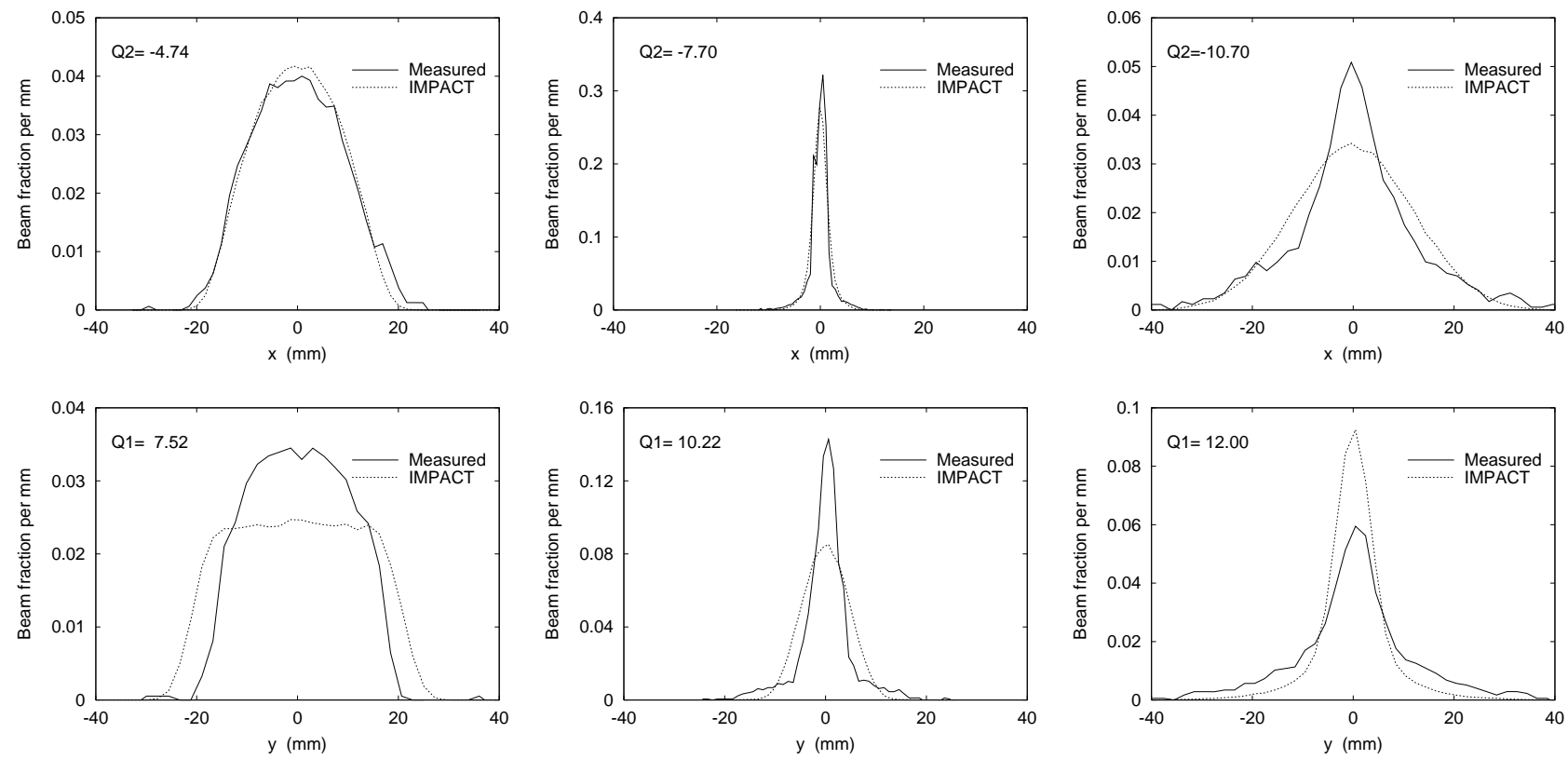

Figure 1: Comparison of measured profiles with IMPACT simulations using RFQ output beam predicted by PARMTEQM. The top row shows $x$ profiles for three values of Q2 and the bottom row shows $y$ profiles for three values of Q1.
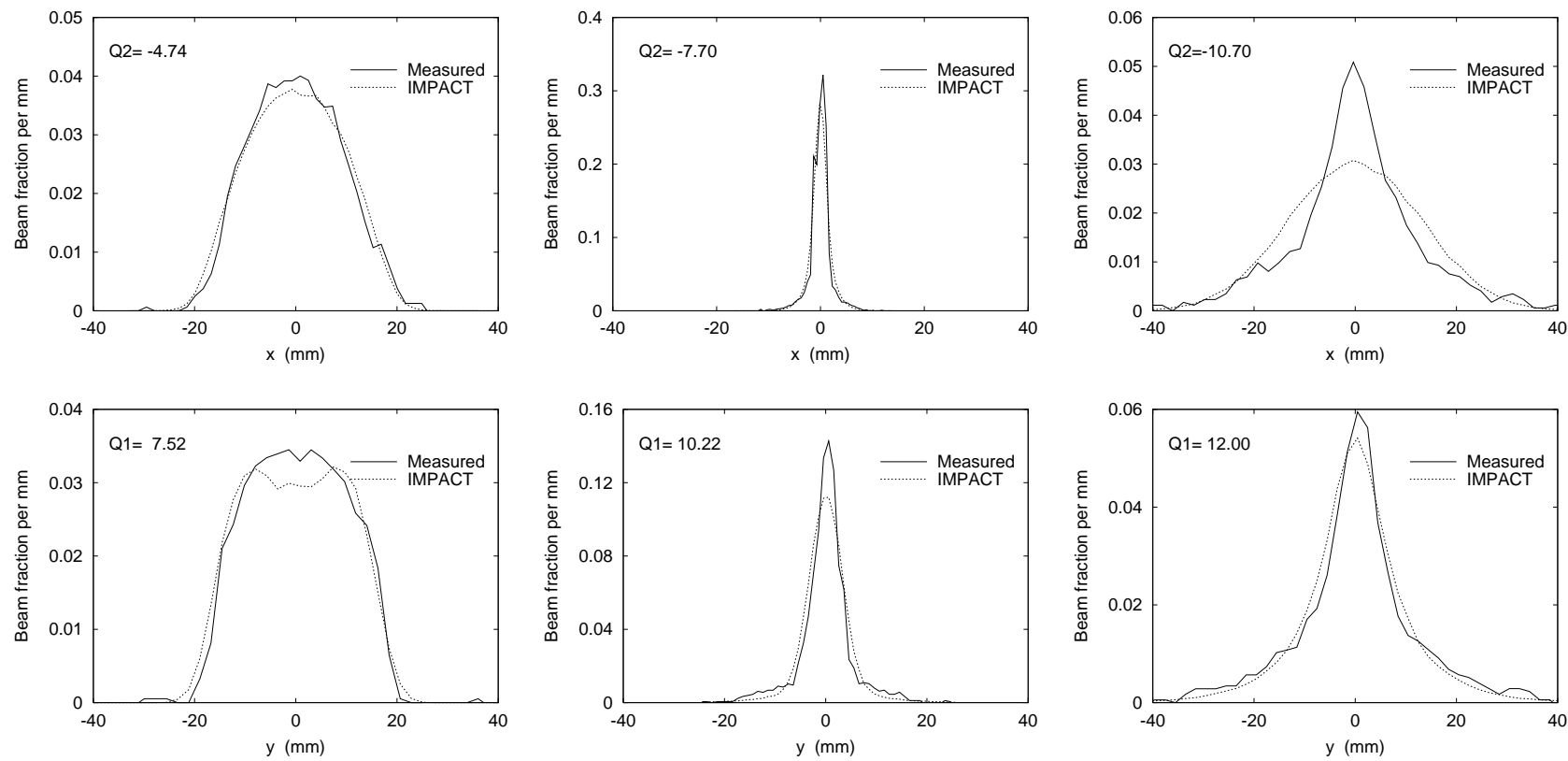

Figure 2: Comparison of measured profiles with IMPACT simulations using RFQ output beam determined by fitting rms widths to LINAC simulations. The top row shows $x$ profiles for three values of Q2 and the bottom row shows $y$ profiles for three values of Q1. 

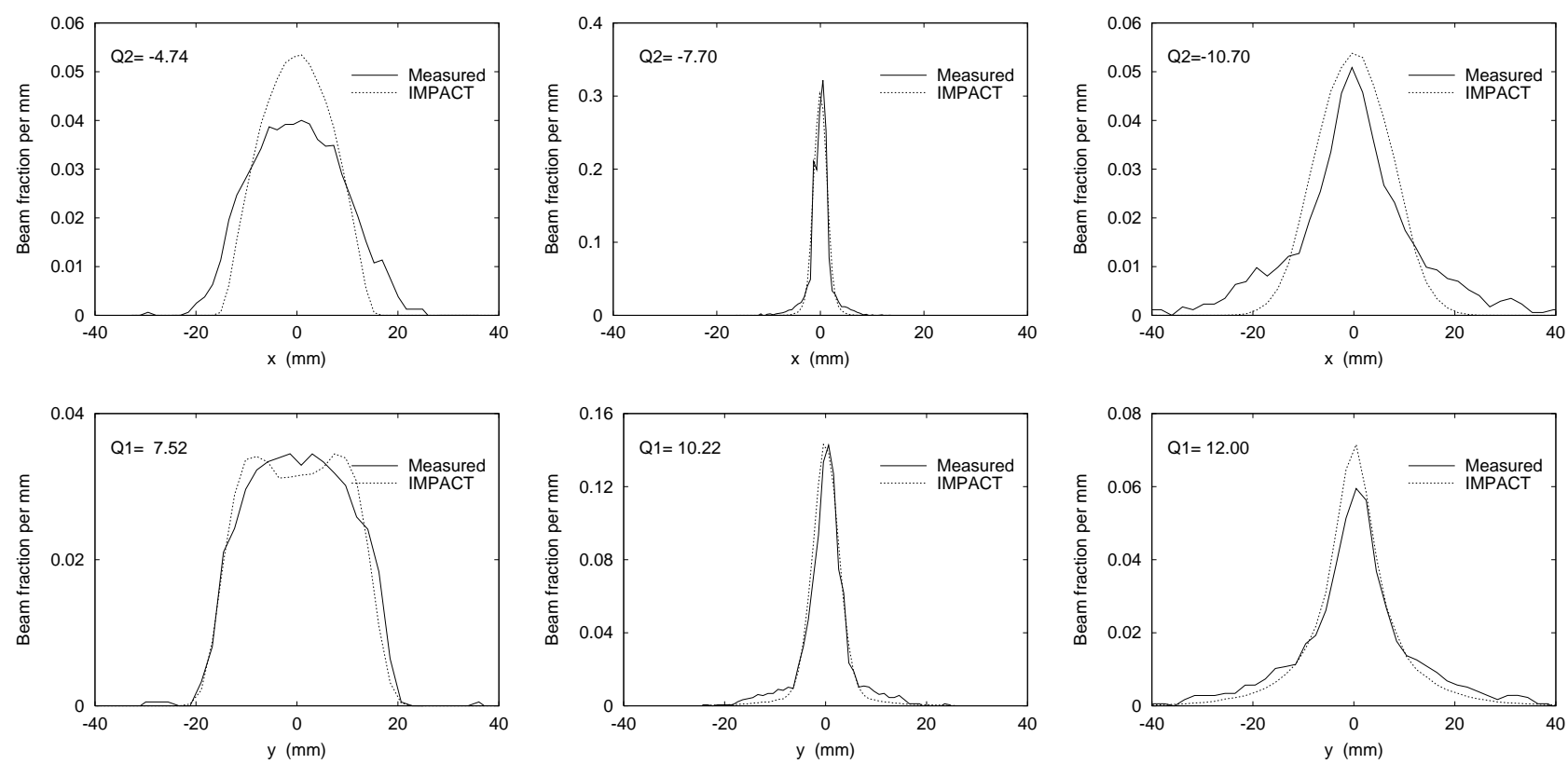

Figure 3: Comparison of measured profiles with IMPACT simulations using RFQ output beam determined by fitting profile shapes to IMPACT simulations. The top row shows $x$ profiles for three values of Q2 and the bottom row shows $y$ profiles for three values of Q1.

Table 1: Courant-Snyder parameters for various RFQ exit beams (unnormalized values). The dashed curves of Fig. 3 correspond to IMPACT fit 1 .

\begin{tabular}{lcccc}
\hline \multicolumn{5}{c}{ Courant-Snyder parameters in $x$ direction } \\
\hline RFQ exit beam & $\alpha_{x}$ & $\begin{array}{c}\beta_{x} \\
(\mathrm{~mm} / \mathrm{mrad})\end{array}$ & $\begin{array}{c}\epsilon_{x} \\
(\mathrm{~mm} \cdot \mathrm{mrad})\end{array}$ & $\begin{array}{c}\sqrt{\beta_{x} \epsilon_{x}} \\
(\mathrm{~mm})\end{array}$ \\
\hline PARMTEQM & 1.59 & 0.398 & 2.03 & 0.899 \\
LINAC fit & 1.79 & 0.358 & 2.11 & 0.869 \\
IMPACT fit 1 & 2.45 & 1.21 & 1.78 & 1.47 \\
IMPACT fit 2 & 2.30 & 1.39 & 1.47 & 1.43 \\
\hline
\end{tabular}

\begin{tabular}{lcccc}
\hline \multicolumn{5}{c}{ Courant-Snyder parameters in $y$ direction } \\
\hline RFQ exit beam & $\alpha_{y}$ & $\begin{array}{c}\beta_{y} \\
(\mathrm{~mm} / \mathrm{mrad})\end{array}$ & $\begin{array}{c}\epsilon_{y} \\
(\mathrm{~mm} \cdot \mathrm{mrad})\end{array}$ & $\begin{array}{c}\sqrt{\beta_{y} \epsilon_{y}} \\
(\mathrm{~mm})\end{array}$ \\
\hline PARMTEQM & -2.74 & 0.726 & 2.04 & 1.48 \\
LINAC fit & -2.48 & 0.892 & 2.62 & 1.53 \\
IMPACT fit 1 & -3.54 & 1.61 & 1.38 & 1.49 \\
IMPACT fit 2 & -3.40 & 1.19 & 1.80 & 1.46 \\
\hline
\end{tabular}

predicted or LINAC-fit beam. Also shown in Table I is another IMPACT fit (labeled fit 2), obtained using different starting values in the nonlinear optimization. Both IMPACT fits are about equally good in terms of the least-squares errors (not shown) but correspond to quite different emittance values (and the other Courant-Snyder parameters).

\section{DISCUSSION}

Fitting with a 3-D nonlinear space charge code, using all the data instead of just the rms widths, still did not result in simulated profiles that looked more like the measurements for the larger quadrupole gradients. There is a problem with our model. Some physics is missing in our simulations or the beam coming from the RFQ has some additional structure that cannot be described by the three Courant-Snyder parameters. Space-charge effects are significant only just downstream of the RFQ exit; it is unlikely that the very asymmetrical beam in the HEBT is generating the strange profile shapes. This has been verified by studies using IMPACT and the TRACE 3-D code.

Table I also shows the value of the quantity $(\beta \epsilon)^{1 / 2}$ for the various beams. These are the rms beam sizes at the RFQ exit. These quantities are nearly the same for all the IMPACT fits, even though they have quite different emittance values. It appears that the quad scans may be better at measuring the beam size at the RFQ exit than emittances. Clearly, we have to resolve the discrepancy between the measured and simulated profiles to get good fits. An improved model may result in more accurate emittance measurements.

\section{REFERENCES}

[1] W.P. Lysenko, et al., "Determining Phase-Space Properties of the LEDA RFQ Output Beam," Proceedings of the XX International Linac Conference, Monterey, CA, USA, August 21-25, 2000, pp. 809-811.

[2] M.E. Schulze, et al., "Beam Emittance Measurements for the Low-Energy Demonstration Accelerator Radio-Frequency Quadrupole," Proceedings of the XX International Linac Conference, Monterey, CA, USA, August 21-25, 2000, pp. 575-577. 